\title{
Fahr's Disease: A Rare Neurological Disease Frequently Misdiagnosed as Acute Psychosis, or Mood Disorder
}

\author{
Suneel Kumar ${ }^{1}$, Khalid Sher ${ }^{1}$, Saeed Ahmed ${ }^{2}$, Savita Naik ${ }^{2 *}$, Shahana Ayub ${ }^{3}$, Fatima B Motiwala² and Rizwan Ahmed
}

${ }^{1}$ Department of Neurology, Jinnah Post Graduate Medical Centre, Karachi, Pakistan

${ }^{2}$ Department of Psychiatry, NYU School of Medicine, New York, NY, USA

${ }^{3}$ Agha Khan University Hospital, Karachi, Pakistan

\begin{abstract}
Fahr's disease is a rare, degenerative neurological disorder characterized by idiopathic symmetrical and bilateral intracranial calcification. We present a case of 32 years old man with Fahr's disease who presented with episodes of inappropriate excessive laughing, generalized tonic clonic seizures associated with loss of consciousness, frothing from mouth, urine incontinence and difficulty in walking due to neurological deficit. MRI of brain demonstrated calcification of bilateral basal ganglia, thalami and left dentate nucleus of left cerebellum. The clinical presentation similar to our patient is frequently misdiagnosed due to identical manifestation to other neurological and psychiatric disorders especially in young to middle aged individuals. Fahr's disease may often misdiagnosed as a patient of acute psychosis or epileptic seizures.
\end{abstract}

\section{Introduction}

Fahr's disease was first described by German neurologist Karl Theodor in 1930. It is considered as a familial or sporadic disorder. Majority of cases have been reported to have autosomal dominant inheritance, likewise very few cases are also reported to have autosomal recessive inheritance. Fahr's disease is also known as familial cerebral ferrocalcinosis or Familial idiopathic basal ganglia calcification (FIBGC). It is characterized by abnormal deposition of calcium in the areas of brain that control body movement, commonly involves basal ganglia, dentate nuclei, putamen, thalami and cerebellum. Involvement of these areas clinically presents with extrapyramidal symptoms, psychiatric manifestations, cerebellar symptoms with gradually progressive cognitive impairment. The onset of disease is insidious, typically the age at onset of clinical presentation is in middle age as in our case, and onset of early age is frequently misdiagnosed as a psychiatric illness [1]. Presentation of disease could vary with the age and course of illness. The clinical features usually include psychosis, cognition impairment, and symptoms of mood disorders, epileptic seizures and dementia [2,3]. Excessive extensive intracranial calcification is also associated with psychiatric manifestation [3]. Diagnosis of Fahr's disease is based on combinations of clinical features, brain imaging and exclusion of other causes of intracranial calcifications [4], there is absence of standard course of treatment for this illness, usually patients are treated according to symptomatology.

\section{Case Report}

32 year unmarried male presented to our tertiary care hospital, with complaints of repeated generalized tonic clonic seizures since four years. During these episodes of seizures were associated with loss of consciousness, frothing from mouth and urine incontinence. He had difficulty in walking for eight years, that is progressive in nature. In addition to that, his family described a change in his overall behavior like; bouts of disorientation, forgetting his way to home, irritability, and episodes of inappropriate laughing. On neurological examination, patient was alert, oriented to time, person and place, cranial nerves, power, sensation found normal. Cerebellar signs including finger nose, heel shin test, in coordination were positive. Assessment of his cognitive level included mini-mental status examination score of 22/30.

In order to exclude other differential that may lead to secondary intracranial calcification, patient went through series of blood tests, that included, complete blood picture, Erythrocyte sedimentation rate, iron studies, Serum calcium and phosphate level, Serum parathyroid hormone level, thyroid hormone levels, serum creatinine, serum ceruloplasmin and urinary copper. Results of these tests in addition to Serum lactate and pyruvic acid, serological tests for syphilis and $\mathrm{HIV}$, and CSF study to rule out other metabolic, infectious cause, HIV encephalitis and SLE appeared normal. His Electroencephalogram (EEG) and Electrocardiogram (ECG) were also done and showed normal.

Moreover to exclude other possible brain pathologies, patient was scheduled for brain imaging tests. Prior to investigate through imaging techniques, symptomatic treatment was started with one of antipsychotics Quetiapine to address his behavioral symptoms, similarly he was also started on sodium valproate for repeated seizures. MRI brain plain (T1\& T2 weighted axial and FLAIR coronal images were obtained), hyperintense on T1W and hypointense on T2W images bilateral basal ganglia, thalami and left dentate nucleus of left cerebellum showed calcification that is consistent with presence of Fahr's syndrome (Figures 1 and 2). On the basis of clinical history, laboratory investigations and brain image, we diagnosed this patient for Fahr's disease. Patient gradually responded to antipsychotics and antiepileptic therapy and currently his behavioral symptoms are absent and seizures are well controlled.

\section{Discussion}

In 1930, Karl Theodor Fahr reported a first report of fahr's disease, it was a case of 10 years of boy with title of "Idiopathic Calcification the

*Corresponding author: Savita Naik, Department of Psychiatry, NYU Schoo of Medicine, 145 East 32 ${ }^{\text {nd }}$ street, New York, USA, Tel: 201-687-6749; E-mail: savitanaik2000@gmail.com

Received July 19, 2013; Accepted August 24, 2013; Published August 27, 2013

Citation: Kumar S, Sher K, Ahmed S, Naik S, Ayub S, et al. (2013) Fahr's Disease A Rare Neurological Disease Frequently Misdiagnosed as Acute Psychosis, or Mood Disorder. J Neurol Disord 1: 130. doi:10.4172/2329-6895.1000130

Copyright: (C) 2013 Kumar S, et al. This is an open-access article distributed under the terms of the Creative Commons Attribution License, which permits unrestricted use, distribution, and reproduction in any medium, provided the original author and source are credited. 


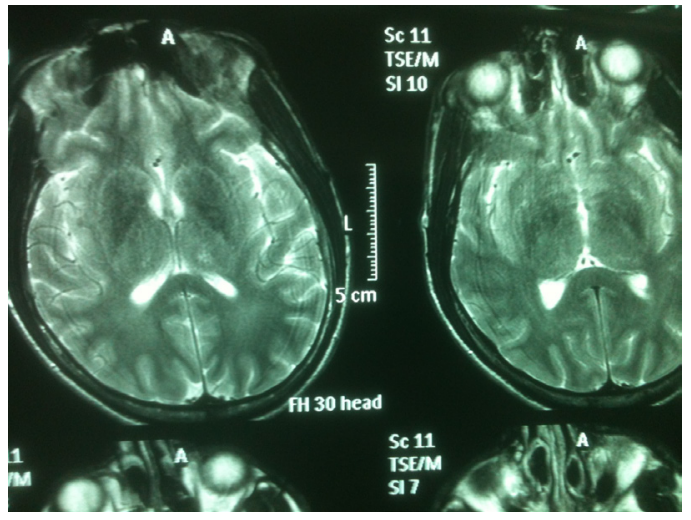

Figure 1: MRI Brain: Calcification of bilateral basal ganglia, thalami and left dentate nucleus of left cerebellum.

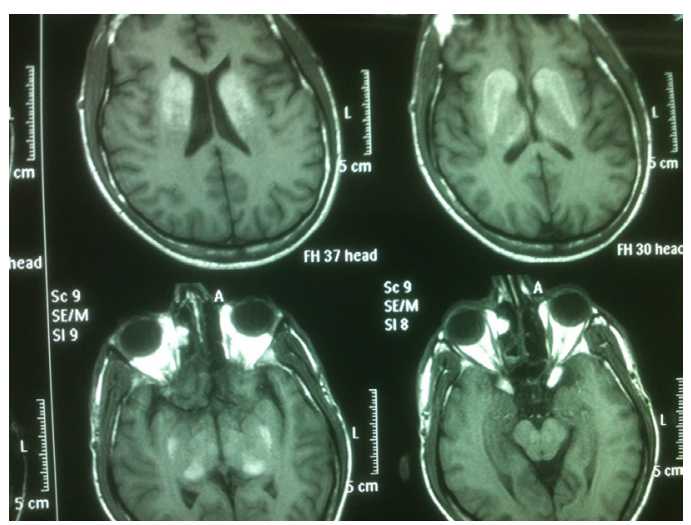

Figure 2: MRI Brain: Calcification of bilateral basal ganglia, thalami and left dentate nucleus of left cerebellum.

cerebral vessels". This disease is not well recognized with less common names Chavany-Brunhes syndrome and Fritsche's syndrome. Fahr's Disease is quiet most commonly used term for this illness. Published literature is not sufficient to provide exact prevalence of this rare disease. Similarly it is also very difficult to predict prognosis of Fahr's disease in absence of evidences regarding correlation between age, extent of calcium deposition, or outcome based on areas of brain are involved. Two forms of Fahr's disease have been classified in the literature according to possible etiology [5]. Primary form is familial or sporadic as in our case. In familial category, autosomal dominant is more common than recessive one. Secondary form is caused by other metabolic or endocrinological disorders such as hypoparathyroidism, hyperpara-thyroidism or pseudohypoparathyroidism [6]. Our patient was not well aware of his family history related to Fahr's disease, therefore familial predisposition can neither be excluded nor well documented in this case [7].

In patients where the positive family history cannot be documented as in our case, following five criteria should meet in diagnosis of Fahr's disease:

- Neuroimaging showing bilateral calcification of the basal ganglia or other brain regions

- Neuropsychiatric manifestations and/or progressive neurological dysfunction
- Although it can also present earlier in life, onset of symptoms occurs typically in the fourth or fifth decade of age.

- Absence of other causes for intracranial calcification such as biochemical abnormalities and somatic features suggestive of a mitochondrial or metabolic disease or other systemic disorder

- Absence of an infectious, toxic, or traumatic cause

Diagnosis of Fahr's disease could be challenging, may be due discrepancy between the clinical manifestations and imaging findings. A patient with classical clinical presentation could discrepant with poor imaging results and vice versa with good imaging results. Whereas clinical presentation of our case report is typically consistent with imaging results, moreover our patient presented with more rare entity with compromised memory as he had difficulty to find his home. The present case explains that Fahr's Disease may present without commonly presenting movements disorder but with prominent seizures, cognitive and behavioral abnormalities. Likewise this patient has distinguished clinical picture similar to a case, reported by Benke et al. [8] that showed frontal lobe syndrome and dementia that makes it worth to point it out.

To date there is no proven treatment documented for Fahr's disease. Syoichiro Kono et al. [9] reported a case of Fahr's disease presenting chorea successfully treated by the use of quetiapine. This case explains the effectiveness of quetiapin and sodium valproate in controlling behavioral problems and seizures respectively associated with Fahr's disease.

\section{References}

1. Srivastava S, Bhatia MS, Sharma V, Mahajan S, Rajender G (2010) Fahr's disease: An incidental finding in a case presenting with psychosis. Ger J Psychiatry. 13: 86-90

2. Modrego PJ, Mojonero J, Serrano M, Fayed N (2005) Fahr's syndrome presenting with pure and progressive presenile dementia. Neurol Sci 26 367-369.

3. König P (1989) Psychopathological alterations in cases of symmetrical basa ganglia sclerosis. Biol Psychiatry 25: 459-468.

4. Lam JS, Fong SY, Yiu GC, Wing YK (2007) Fahr's disease: a differential diagnosis of frontal lobe syndrome. Hong Kong Med J 13: 75-77.

5. Manyam BV (2005) What is and what is not 'Fahr's disease'. Parkinsonism Relat Disord 11: 73-80.

6. Shoyama M, Kitabata Y, Kaku T, Shinosaki K (2005) Evaluation of regiona cerebral blood flow in fahr disease with schizophrenia-like psychosis: a case report. AJNR Am J Neuroradiol 26: 2527-2529.

7. Lazar M, Ion DA, Streinu-Cercel A, Badarau Al (2009) Fahr's syndrome: diagnosis issues in patients with unknown family history of disease. Rom $J$ Morphol Embryol 50: 425-428.

8. Benke T, Karner E, Seppi K, Delazer M, Marksteiner J, et al. (2004) Subacute dementia and imaging correlates in a case of Fahr's disease. J Neurol Neurosurg Psychiatry 75: 1163-1165.

9. Syoichiro Kono, Yasuhiro Manabe, Tomotaka Tanaka, Daiki Fujii, Yasuko Sakai, et al. (2009) A Case of Fahr's Disease Presenting as Chorea Successfully Treated by the Use of Quetiapine; Clinical Medicine: Case Reports 263-265. 